\title{
Trombosis Venosa Profunda en el Síndrome Nefrótico
}

\author{
Dra. M. Angela Delucci B.1; Dr. Eric Solar G.2; Dra. Edda Lagormarsino F. 3; \\ Di. Iternán Taboada 1.4 ; Dr. J. Domingo Arce V.5
}

\section{Deep Venous Leg Thrombosis in a Boy with Nephrotic Syndrome}

\begin{abstract}
Thrombosis is a rare complication in patients wit l nephrotic syndrome, and scant coagulation data is available in this disorder. An jlfustrative case of peripheral deep venous thrombosis of the left leg attributed to the hypercoagulable state of the nephrotic syndrome in a 12 years old boy with minimal glomerular lesions begining at the age of eight years is discussed. An important decrease in antithrombin (II together with high blood fibrinogen contentration and increased number of thrombocytes were demostrated in tilis patient. Possible patlogenesis and treatment with Vitamin $K$ antagonists are discussed.

(Key words: Nephrotis Syndrome. Venous Thrombosis. Hypercoagulable State. Vitamin K Antagonists).
\end{abstract}

El Sindrome Nefrótico (SN) se define como una entidad clínica caracterizada por proteinuria significativa (mayor que $100 \mathrm{mg} \times \mathrm{kg} \times 24 \mathrm{hrs}$ ). hipoproteinemia e hiperlipemia. En 1827, Bright ${ }^{1,2}$ describió por primera vez una enfermedad renal asociada a edema y albuminuria. Diez años más tarde se publicó un caso dc tromboembolismo en un paciente con SN con trombosis de la vena rena ${ }^{3}$. Sin embargo no fue hasta 1954 en que se señaló la existencia de mayor riesgo de trombosis vasculares en los pacientes on SN. Desde entonces han aparecido numerosas publicaciones que exploran factores predisponentes y complicaciones trombóticas, especialmente de las venas renales. ${ }^{5,6}$

A propósito de un caso de SN con trombosis venosa profunda de una extremidad inferior, estudiamos los factores de coagulación implicados y presentamos las alternativas de tratamiento. No hemos encontrado referencias nacionales sobre la materia.

1. Médico Pediatra. Servicio de Pediatría. Hospital Sótero de] Río. SSMSO.

2. Médico Nefrólogo. Servicio de Pediatría. Hospital Sótero del Río. SSMSO.

3. Profesor Adjunto de Pediatría, Universidad Católica. Servicio de Nefrología Infantil. Hospital Sótero del Río. SSMSO.

4. Profesor Adjunto de Pediatría, Universidad Católica. Servicio de Hematología Infantíl. Hospital Sótero del Río. SSMSO.

5. Médioo Radiólogo. Servicio de Radiología. Clínied Dárila.

\section{Caso Clínico}

Varón de 12 años, portador de daño cerebral difuso, secundario a hipoxia perinatal. En Febrero de 1983 se le diagnosticó $\mathrm{SN}$, porque tenia edema generalizado, proteinuria de $300 \mathrm{mg} \mathrm{x} \mathrm{kg}$ $x 24 \mathrm{~h}$; proteinemia total $3,9 \mathrm{gr} \%$, albúmina plasmática de $1,9 \mathrm{gr} \%$; colesterolemia $456 \mathrm{mg} \%$; creatinina plasmática $0,82 \mathrm{mg} \%$; sedimento de orina normal. La biopsia renal mostró, en la microscopia óptica, lesiones minimas. La inmunofluorescencia del tejido renal fue negativa para $\mathrm{C}_{3}, \mathrm{C}_{4}, \mathrm{C}_{1} \mathrm{q}, \mathrm{Ig} \mathrm{A}, \mathrm{Ig} \mathrm{E}$ y fibrinógeno; $\lg \mathrm{G}, \mathrm{Ig} \mathrm{M}$ Jevemente positivas en forma difusa en la zona mesangio capilar. Se trató con prednisona y la respuesta fue buena.

lngresó al Servicio de Pediatría el 27 de Febrero de 1985 con edema facial, ascitis, diuresis de $0,4 \mathrm{ml} \times \mathrm{kg} \times \mathrm{h}$, normotenso, con proteinuria de $245 \mathrm{mg} \times \mathrm{kg} \times 24 \mathrm{~h}$, sin hematuria. Se le trató con prednisona, $2 \mathrm{mg} \times \mathrm{kg} x$ día, oral, obteniéndose buena respuesta diurética y desaparición del edema a los 23 días de tratamiento. Estado con tratamiento esteroidal intermitente sufrió un episodio de diarrea aguda, durante el cual se observó, en la extremidad inferior izquierda, un aumento brusco de volumen, frío, indoloro sin cianosis: Mediante Doppler se detcctó la presencia de flujo arterial. En la flebografía se encontró evidencia de trombosis venosa profunda (Figura 1). El recuento de plaquetas en la sangre fue $485 \times 10^{3} \mathrm{x} \mathrm{mm}^{3}$ (V.N.: 200 a 400 x $10^{3}$ ) fíbrinógeno $710 \mathrm{mg} \%$ (V.N.: 150 a 600 $\mathrm{mg} \%$; antitrombina IIl efectuada por inmunoelectroforesis: $0,21 \mathrm{ug} x \mathrm{ml}$ (V.N.: 0,18 a $1,3 \mathrm{ug}$ $\mathrm{x} \mathrm{ml}$ ): antitrombina III, por método de coagula- 


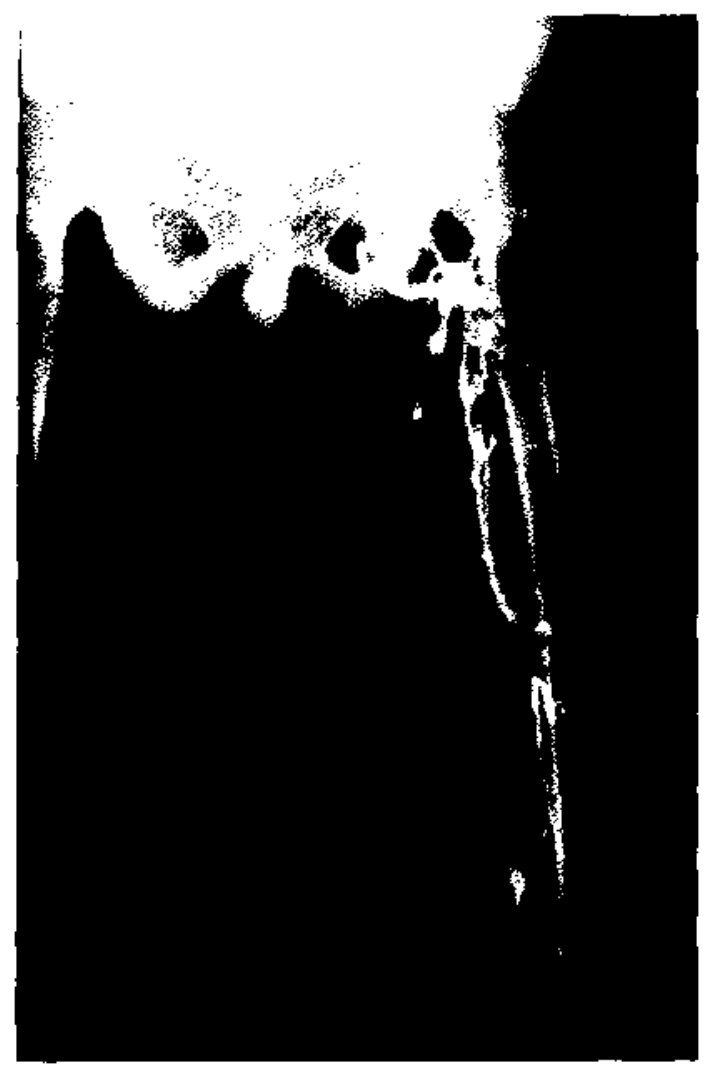

Figura 1: linbotrombosis dol sisumá venoso profundo del muslo y de las venas ilíacas.

ción de Von Kaulia de 7.4" (V.N.: 19" - 69"), factor V: 100\%; factor VIII: 68\%; TTPK: 27,2\%: (V.N.: $20^{\prime \prime}-30 "$ ); Protrombina: $100 \%$.

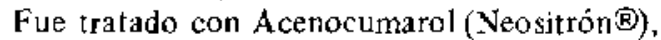
antagonista de la Vitamina $\mathrm{K}$. manteniendo la actividad de protrombina entre 15 y $20 \%$ de 10 normal, con lo que se observó desparación de los sintomas en 5 dias. Quince días después, la protrombina bajó a $8 \%$, el paciente sufrió epistaxis y hematuria; fue necesario suspender el tratamiento $y$ administrar Vitamina $\mathrm{K}$ y plasma fresco con lo cual cesaron las hemorragias. El paciente fue dado de alta sin edema, con proteinuria de $48 \mathrm{mg} \times \mathrm{kg} \mathrm{x} 24 \mathrm{~h}$, y pruebas de coagulación en rangos normales, en tratamiento esteroidal.

\section{DISCUSION}

La trombosis venosa profunda, a pesar de ser una complicación poco frecuente en el SN, debe tenerse presente ya que puede ser fatal, si ocurre en territorios como venas o arterias pulmonares, venas o arterias periféricas profundas (renales, coronarias, pulmonares, mesentéricas, etc.). $\mathrm{Mu}$ - chos de estos accidentes son diagnosticados tardiamente en la necropsia, debido a su räpida instalación y a sus escasos sintomas. ${ }^{7-10}$ Los mecanismos fisiopatológicos implicados en el estado de hipercoagulabilidad de estos pacientes, se basat en la existencia de numerosas anomalias en la hemostasia, entre ellas trombocitosis, aumento del fïbrinógeno con disminución del plasminógeno, elevación de los factores V. VI, VIII y $X$ que aceleran la formación de trombina y la disnuinución de antitrombina IIl (A.T. III) $9.1 \mathrm{~J}$

En el SN la concentración plasmática de las proteinas del sistema hemostático cstá en función del peso molccular y de su capacidad de sintesis. Las proteínus de alto peso molecular. que no filtran hacia la orina estan elevadas, y las de peso molecular menor se encuentran en concentraciones bajas, como es el caso del plaminogeno, la antitrombina III (A.T. III) $y$ ta $\alpha$ 1 -antitripsina. La concentración de los factores Vitamina $K$ dependientes es normal. La relación precisa entre estas anomalías y la trounbosis aún no es clara. El factor principal parece ser el déficit de antitrombina III cuya principal fun* ción es neutralizar la actividad trómbica plasmatica en el sujeto normal. Kauffinan y cols. ${ }^{12}$ demostraron una relación directa entre el déficit de A.T. IIL y la frecuencia de complicaciones tromboembólicas. El aumento de or 2 macroglobulina juega un rol antagónico en la mayoría de los S.N. lo que explica que a pesar de la baja concentración de A.T. III, la actividad antitrómbica global del plasma en estos pacientes sea superior a la de los normales. Sería en los casos donde la actividad de $\propto 2$ macroglobulina no está tan aumentada donde se presentan las trombosis.

La A.T. III, previene el depósito inapropiado de fibrina en los vasos, y su déficit favoreceria el tromboembolismo. Este hecho se confirma al describi en varias familias déficit congénito de A.T. III, Jos que presentan problemas trombóti$\cos ^{9}$. Estudios en pacientes con S.N. que relacionan fenómenos trombóticos y niveles de A.T. III, demostraron que el $90 \%$ de los pacientes que presentaban trombosis tenían valores de A.T. III bajo el $70 \%$ de lo normal ${ }^{11}$.

Existe una correlación negativa entre los valores de A.T. III y la excreción de proteinas urinarias, y una relación positiva cntre niveles plasmáticos de A.T. III y la albuminemia. Cuando la concentración de albúmina plastnática cae bajo $2 \mathrm{gr} \%$ aumentan las complicaciones tromboembólicas.

Otro factor que participa es la disminución del plasminógeno, secundaria a plasminogenuria. La plasmina, forma enzimáticamente activa del plasminógeno es responsable de la proteólisis del 
coägulo de fibrina. De esta manera la actividad fibrinolítica alterada, predispone al tromboembolismo. ${ }^{13}$ A pesar de que la alteración en los lipidos plasmáticos es un hecho importante en el $S . N$., la relación entre aumento de trombosis e hipertrigliceridemia con hipercolesterolemia es incierta. Los cambios en los lípidos plasmáticos pueden alterar las propicdades de los lípidos de la membrana plaquetaria, causando un aumento de Ia agregación en presencia de adenosín difosfato (A.D.P.). Estos cambios cualitativos se han observado en pacientes con hipercolesterolemia y S.N. ${ }^{14}$ Alteraciones en los lípidos también pue. den acelerar la generación de tromboplastina y deprimir el potencial fibrinolítico. El empleo de estercides puede contröbuir a los fenómenos trombóticos del S.N. Cosgriff describió aumento en la frecuencia de episodios tromboembólicos durante la terapia esteroidal ${ }^{15}$. Ozsoylu y cols. ${ }^{16}$ sugieren que los corticoides inducirian aumento del factor VIII y de otras proteinas séricas, que favorecen el estado de hipercoagulabilidad. Liberman ${ }^{17}$ menciona una dismisución de la actividad fibrinolítica durante el tratamiento esterotdal y sugiere que existiría una lisis incomplcta del trombo, favoreciendo de esta manera un aumento en el depósito de fibrina y plaquetas. La hipertensión, la hipovolemia, los complejos inmunes circulantes y la susceptibilidad a infecciones han sido implicadas en la génesis del estado de hipercoagulabilidad, desconociéndose en qué forma contribujían cada una de Ellas. 18,19

Las alteraciones de la coagulación encontradas en el S.N. podrian estar relacionadas con el tipo de lesión histológica. Las trombosis de vena rena] se han visto asociadas en alrededor del $50 \%$ a la glomerulonefritis membranosa, aunque no hubieran sintomas clínicos de trombosis, porque la oclusión de la vena renal puede ocurrir en forma lenta, permitiendo el desarrollo de un extenso sistema venoso colateral.

Para modificar la incidencia de complicaciones tromboembólicas en el S.N. se ha sugerido el uso de heparina como anticoagulante. ${ }^{7,9}$ Se sabe que la antitrombina III, deficitaria en el S.N., es cofactor de la heparina. El aumento de las lipoproteínas que fijan en gran parte la heparina, compitiendo con la A.T. III y con el factor 4 (anti heparina) plaquetario, contribuye a la resistencia de este tratamiento. Ademis está descrita la acción inhibidora de la heparina sobre la actividad antitrómbica de la $\propto 2$ macroglobulina. $\mathrm{L}_{a}$ adición in vitro de heparina al plasma de un niño con S.N. disminuye la actividad antitrómbica, agravando la trombosis. Esta hipótesis no está completamente demostrada in vivo, pero la duda es suficiente para recurrir en estos pacientes de riesgo a los antagonistas de la $\mathrm{Vit} \mathrm{K}$, que tienen una acción antiprotombinica. El uso de los antagonistas de la Vit $\mathrm{K}$ conlleva otros problemas por su unión variable con la albúmina y su liberación por el uso de algunos medicamentos, como la furosemida. ${ }^{9.11}$

El tratamiento anticoagulante profiláctico no está indicado, debido a la escasa frecuencia y evolución favorable, cuando la complicación se diagnostica y se trata en forma oportuna. ${ }^{9,20}$

En el tratamiento preventivo de los fenóme nos tromboembólicos deberá evitarse la inmovi. lización prolongada, la hipovolemia y la hemo. concentración producida por un tratamiento diurético inapropiado. La única situación en que el tratamiento anticoagulante preventivo estaría indicado seria el $S N$ resistente a tratamiento, que evoluciona en forma crónica. En estos casos el riesgo del tratamiento anticoagulante prolongado debe ser sopesado con la incidencia y severidad de los fenómenos tromboembólicos y la posibjlidad de que éstos recidiven. 9,20

En nuestro paciente la complicación trom. boembólica se debió, probablemente, a varios factores; entre ellos la inmovilización prolongada por el daño cerebral, un episodio de diarrea aguda que agravó su hipovolemia y el déficit de A.T. LII secundario a su proteinuria. Debe recordarse esta complicación al examinar a un pa. ciente con SN, especialmente si está en etapa de mayor protejnuria, en la fase diurética o inmovilizado, ya que a pesar de ser poco frecuente, tiene una elevada mortahidad.

\section{RESUMEN}

Un escolar de 12 años portador de Síndrome Vefrótico compatible con lesiones histológicas mínimas, sufrió durante su evolución una trombosis venosa profunda de la extremidad inferior izquierda, que fue confirmada por flebografía. Los estudios de coagulación mostraron trombocitosis, hiperfibrinogenemia y disminución de antitrombina III; factores que se han descrito implicados en la génesis de los fenómenos tromboembólicos. Se analizan los posibles mecanismos que producen la coagulación y se discute el uso de antagonistas de la Vitamina $\mathrm{K}$, como tratamiento de esta complicación.

\section{REFERENCIAS}

1. Bright $R_{1}$ : Reports of medical cases, selected with a vicw of illustrating the symptoms and cure of disease by a reference to morbid anatomy. London, Longman, Recs, Orme, Brown and Green, 1, 1827.

2. Print R.D.: Bright's disease: clinical manifestations in relation to etiology and prognosis. N. Engl. J. Med. 235: 674, 1946. 
3. Royer P.P.: Tuaité des maladies des reins, it des alterations de la decretion urinarie. Paris, Chez J,B. Baillière. pag. 269, 1140.

4. Fishberg A.M.: Chronic Nephrosis, hypertension and nephritis Philadelphia, Lea and Febiger, pag. $478,1954$.

S. Andrassy K., Ritz F., Bommer J.: Hipercoagulability in the nephrotic syndrome. Klein Wochenschr. 58: 1029,1980 .

6. Kanfer A., Klemknecht D., Broyer M., et al.: Coagulation studies in 45 cases of nephrotic syndrome without utemia. Tromb. Diath. Haemorrh, 24: 562.1970.

7. Habib R., Courtecnisse V.: Thrombose des artères pulmonaires dans les syndrome néphrotiques. J. Urol. Néphrol. 74: 349, 1968.

8. Loirat $C_{\text {- }}$ Complications extra-rénales du syndrome néphrotique. In: Journées Parisiennes de Pćdiatrje. Flam. Méd.-Scjenc. l: 263, 1973.

9. Wagoner $R$., Stanson A.. Holley $\boldsymbol{K}$. et al.: Renal vein thombosis in idiopathic membranous glomerulopathy and nephrotic syndrome: Incidence and significance. Kidney Int. 23: 368, 1983.

10. Bensman A., Vasmant D., Lasfargnes G.: Manifestatios frustes des thromboses artèrielles pulmonaires an cours du sydrome néphrotique. Arch. Franc. Pédiatr. 40: 335, 1983 .

11. Guillot M., Fischer 4. et ol.: Syndrome néphrotique, deficit en antithrombine [II et thromboses a repeticion. Arch. Franc. Pédiatr. 9: 930, 1979.

12. Kauffmann $R$. Veltkamp $J$., Tilberg $N$. er al.: Asquired antithrombin $\amalg I$ deficiency and throm- bosis in the nephrotic syndrome, Am. J. Med. 65: $607,1978$.

13. Lan S.O., Tkachuch I.Y., Hasegawa D.K. et al.: Plasminogen and antithrombin III deficiencies in the childhood nephrotic syndrome associated with plasminogenuria and antithrombinuria J. Pediatr. 96: $390,1980$.

14. Kendall A.G., Lohmann R.C., Dossetor J.B.: Nephrotic syndrome: a hypercoagulable state. Arch. Int. Med. 127: 1021, 197].

15. Cosgriff $S$.W.: Thromboembolic complications assoniated with ACTH and cortisone therapy. JAMA 147: 924, 1951

16. Dzsoylus, Strauss H.S., Diamond L.K.: Effects of corticosteroids on the coagulation of the blood. Nature 195: 1214, 1962 .

17. Libcrman E., Hanser E., Gilchrist G.S. et al.: Thrombosis nephrosis and corticosteroids therapy. J. Pediatr. ?3: 320, 1968.

18. Bukley B.H., Roberts W.C.: The hedrt in systemic lupus erythematosus and the changes induced in it by corticosteroid therapy: a study of 36 necropsy patients. Am. J. Med. 58: 243, 1975

19. Ooi B.S. Ooi Y.M., Pollak V.E.: Citculating immune complexes in renal vein thrombosis. 11 th. Annual Meeting of the Amcrican Society of Nephrology, New Orleans. La, 1978.

20. Sullivan M., Hotgh D., Usar M.C. et al.: Perispheral arterial thrombosis duc to the nephrotic syndrome: The clinical Spcctrum. South. Med. Journal 76: 1011, 1983. 